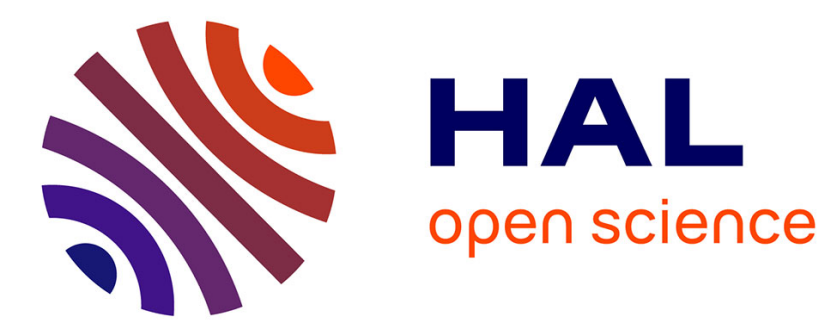

\title{
On defining the notion of complete and immediate formal grounding
}

Francesca Poggiolesi

\section{To cite this version:}

Francesca Poggiolesi. On defining the notion of complete and immediate formal grounding. Synthese, 2016, 193, pp.3147-3167. 10.1007/s11229-015-0923-x . hal-01102067

\section{HAL Id: hal-01102067 \\ https://hal.science/hal-01102067}

Submitted on 13 Jan 2015

HAL is a multi-disciplinary open access archive for the deposit and dissemination of scientific research documents, whether they are published or not. The documents may come from teaching and research institutions in France or abroad, or from public or private research centers.
L'archive ouverte pluridisciplinaire HAL, est destinée au dépôt et à la diffusion de documents scientifiques de niveau recherche, publiés ou non, émanant des établissements d'enseignement et de recherche français ou étrangers, des laboratoires publics ou privés. 


\title{
Francesca Poggiolesi
}

\section{On defining the notion of complete and immediate formal grounding}

\begin{abstract}
The aim of this paper is to contribute to the recent debate on the notion of grounding. More precisely, we will focus on the notion of complete and immediate formal grounding and we will provide a definition of it through the concepts of derivability and complexity. We will show that this definition allows us a subtle and precise analysis of the concept of grounding in several paradigmatic cases.
\end{abstract}

\section{Introduction}

Consider the following sentences

1. In Europe well-functioning thermometers are higher in summer than in winter because in Europe it is warmer in summer than in winter.

2. The action is wrong because it was performed with the sole intention of causing harm.

3. The ball is red and round because the ball is red and the ball is round.

Each of these sentences contains the expression because and each of them can be divided into an antecedent, i.e. what comes after the because ("in Europe it is warmer in summer than in winter", "the action was performed with the sole intention of causing harm" and "the ball is red and the ball is round"), and a consequent, i.e. what comes before the because ("in Europe well-functioning thermometers are higher in summer than in winter", "the action is wrong", "the ball is red and round", respectively). In each case not only can we say that the consequent follows from the antecedent, but also, and most importantly, that the consequent is determined, or explained or accounted for the antecedent. In other terms, in each of the sentences listed above, the antecedent constitutes the reason why or the ground of the truth of the consequent.

Sentences 1-3 are sentences standardly used to exemplify the concept of grounding, which is a concept that has been receiving impressive and increasing interest in contemporary philosophical and logical literature (e.g. [5, 10, 11, 15, $18,19])$. Grounding is often described as an objective relation amongst truths that is explanatory in nature. The linguistic expression because represents one way of conveying the grounding concept; another common way of conveying this concept is given by the expression in virtue of. 
Several scholars have stressed the importance of the grounding concept for philosophical inquiry. Correia and Schnieder [8] clearly state that "it is a phenomenon of the highest philosophical importance"; Fine [12] goes even further and claims "Once the notion of ground is acknowledged, then I believe that it will be seen to be of general application throughout the whole of philosophy." This paper aims to contribute to the debate concerning this notion.

Our starting point will be a distinction, first introduced by a milestone in the history of the study of grounding, namely the Bohemian thinker Bernard Bolzano, between material and formal grounding. While material grounding is a relation that "depends on the particular character of the ideas involved" [21, p. 266] (our translation), formal grounding is a relation that overlooks such a particular character and only "relies on the form of the propositions involved" [21, p. 266] (our translation). Thus, while sentences 1 and 2 are examples of material grounding, sentence 3 is an example of formal grounding.

In this paper we do not aim to analyze both material and formal grounding; rather, we will restrict our attention to the concept of formal grounding. Our goal is to give a definition of this notion. With respect to such a goal, we would like to emphasize two things. The first concerns its originality. In the recent literature on grounding, we can either find discussions on the properties that grounding satisfies (e.g. see $[10,15])$ or about the very existence of the notion of grounding (e.g. see [9]), but, as far as we know, little has been said on how to define such a concept (Bolzano [2] represents a great exception to this trend and we will actually use some of his brilliant intuitions). The second thing to note is the importance of such a goal. Not only it seems crucial per se to have a definition of formal grounding, but also, such a definition could open a new perspective on how to develop a logic of formal grounding.

The paper will be organized as follows. In Section 2 we will introduce classical logic as the background framework of our research and then investigate the relationship between the classical notion of derivability and formal grounding. Section 3 will serve to introduce the concept of complete and immediate formal grounding that will be the specific object of our study; in Section 4 we will examine the relationship between grounding and complexity and introduce a precise definition of complexity. While Section 5 will be used to analyze the case of disjunction and argue for the need for another element, other than derivability and complexity, in the definition of formal grounding, Section 6 will serve to finally state our definition of complete and immediate formal grounding. In Section 7 we will outline some differences between our approach to the concept of grounding and the most prominent approaches (see $[7,13,20]$ ) in the contemporary literature. Finally, in Section 8, we will draw some conclusions and depict some lines of future research. 


\section{Classical logic, derivability and formal ground- ing}

In what follows classical logic will be the framework where we will develop our research on the notion of formal grounding.

Definition 2.1. The language $\mathcal{L}^{c}$ is composed of a denumerable stock of propositional atoms $(p, q, r, \ldots)$, the logical operators $\neg, \wedge$ and $\vee$ and the parentheses $($, ). Formulas are constructed as usual and the set of well-formed formulas of $\mathcal{L}^{c}$ will be denoted by $\mathcal{F}$.

Semantically, classical logic is defined by means of the standard truth-tables containing the two truth values, truth and false. Syntactically, by relying on the means of the natural deduction calculus (e.g. see [23]), we can easily introduce the notion of derivability which is denoted with the symbol $\vdash$. $M \vdash A$ stands for: there exists a natural deduction derivation $d$ from the multiset of formulas $M$ to the formula $A$. The theorems of soundness and completeness (e.g. see [23]) show a perfect correspondence between the semantic and the syntactic perspectives.

Here we are interested in understanding the link between classical derivability and formal grounding. Even at the first glance, the concept of grounding and the concept of derivability seem to be closely connected. But what exactly does their relationship amount to? Certainly, that A is derivable from the multiset of formulas $M$ does not imply that $\mathrm{M}$ ground $\mathrm{A}$. To see this, consider the case of conjunction. While $A$ is derivable from $A \wedge B$, nobody would ever say that $A \wedge B$ is the ground of $A$. What about the inverse then? If $\mathrm{M}$ formally ground A, does this imply that $\mathrm{A}$ is derivable from M? According to Bolzano, this is the case. Formal grounding is grounding amongst propositions which are also derivable:

Let's see this: Bolzano distinguishes between material and formal grounding. The second notion, formal grounding, is grounding between propositions which are also derivable and it is defined as a special kind of derivability. [1, p. 197, italics ours]

Thus for Bolzano formal grounding is a special type of classical derivability. This claim is extremely important for our work. Indeed, if we seriously think that formal grounding is a special sort of classical derivability, then the task of defining formal grounding amounts to the task of finding those criteria that make classical derivability particular. This is precisely what we are going to do in the next sections and what we will focus our attention on. Let us conclude this section by drawing the following general picture, in the light of the observations that we have just made: the background framework of our research is constituted by classical logic, its two truth values and a notion of derivability which typically formalizes proofs by means of which we demonstrate that something is true. On this basis, by imposing certain specific and adequate criteria, we aim to draw out a notion of formal grounding that deals only in truths and that is supposed to represent proofs by means of which we demonstrate why something is true. 


\section{Complete and immediate grounding}

According to what we have said in the previous section, if our aim is to find a definition for the notion of formal grounding, our starting point should be the classical notion of derivability. Now a derivation is usually seen as a tree in which every edge corresponds to an inferential step. Then, even a formal grounding (proof) can be seen as a (special) tree (see $[1,17,22]$ ) in which every edge corresponds to a single grounding step. We are going to call the single grounding step of a grounding tree immediate grounding; while the whole grounding tree will be called mediate grounding. Such a terminology has been firstly introduced by Bolzano [3] and then taken up by Fine [12].

The immediate-mediate distinction is not the only one that we can make concerning the concept of (formal) grounding. There is another central distinction to be drawn which appears to correspond to what Bolzano called complete and partial grounding and which is related to what is referred to nowadays as the difference between full and partial grounding.

According to Fine [12] $\mathrm{A}$ is a partial ground of $\mathrm{C}$ if $\mathrm{A}$ on its own or together with some other truths is a ground of $\mathrm{C}$. Thus, given that $\mathrm{A}$ and $\mathrm{B}$ are the full ground of $A \wedge B$, each of $A$ and $B$ will be a partial ground of $A \wedge B$. The notion of full ground is never explicitly defined but we can take it as $A$ is a full ground of $C$ if the truth of $A$ is sufficient to guarantee the truth of $C$. Bolzano's distinction between complete and partial ground is slightly different. Following the analysis of Sebestik [21] and Tatzel [22, p.13], for Bolzano the (multi)set of all, and only, those truths each of which contributes to ground the truth $C$ is a complete ground of $C$. On the other hand, each of the truths that compose the complete ground of $C$ is said to be a partial ground of $C$.

It seems that Bolzano and Fine both attempt to describe a same distinction, although the way they draw the line between the two concepts is different. For Fine a partial ground of a truth $C$ can also be the full ground of $C$, while for Bolzano this can never be the case, since partial and complete grounds are two disjoint concepts [2, p. 268]. Moreover, while for Fine, the full ground of a truth $C$ does not need to correspond to the (multi)set that gathers together all the truths that ground $C$ - for Fine full ground is just a sufficient condition - for Bolzano this is precisely what characterizes the complete ground of a truth $C$.

In what follows we will focus on the concept of complete and immediate formal grounding in the Bolzanian sense; the precise goal of this paper will actually be to give a definition of the notion of complete and immediate formal grounding. (Thus the notions of complete and mediate, partial and immediate and partial and mediate formal grounding are left for future research). This restriction to what might seem a narrow object of study is actually a common strategy in the literature. Indeed, this is very close to what Bolzano himself was doing: he took the concept of complete and immediate formal grounding as central and thought this was the concept to be characterized in the first place. The definition of the other concepts would have followed consequently (see [22]).

A similar remark can be found in Fine. On the one hand Fine claims that 
The notion of immediate ground would appear to give us something genuinely new; and I find it remarkable how strong our intuitions are about when it does and does not hold. [...] It is the notion of immediate ground that provides us with our sense of a ground theoretic hierarchy. [12, p. 51, italics ours $]$

And then he continues with

It is for this reason that pride of place should be given to the full notion in developing an account of ground. [12, p. 50, italics ours]

The agreement of these two illustrious philosophers on the central role that the concept of complete and immediate formal grounding recovers seems to represent a very good reason for focussing on this notion.

\section{Complexity and complete and immediate for- mal grounding}

Let us now get at the heart of the matter by trying to identify those conditions that allow us to pass from classical derivability to the concept of grounding. In particular, since we focus on the notion of complete and immediate formal grounding, we will have to find those conditions that allow us to pass from a singular inferential step to the concept of complete and immediate formal grounding.

In the past as well as in the recent literature on grounding, one of the noted characteristics of this notion is complexity (e.g. see $[1,4,7]$ ). Bolzano was the first to explicitly claim that in a grounding relation the grounds must be less complex than their conclusion ${ }^{1}$. Indeed, if grounding is an objective relation that is explanatory in nature, it seems hard to claim that a truth $B$ is explained by a truth $A$ which is more complex than $B$. Thus, in a grounding chain, complexity decreases as one goes from the conclusion to its grounds. In particular, in the case of complete and immediate grounding, the grounds must be completely and immediately less complex than their conclusion. But what exactly does being completely and immediately less complex mean? Can we have a precise definition of this notion? While intuitively it might seem easy to decide when a truth is (completely and immediately) less complex than another, to draw the formal counterpart of such a notion is not straightforward. The rest of the section will be dedicated to this task. The result will play a crucial role in our definition of complete and immediate formal grounding.

Anyone who is acquainted with classical logic will certainly know that in this framework there already exists a way of counting the complexity of a formula $A$, and a notion, that of subformula, that might be seen as describing the relation of being less complex. So one might think that we actually already have the

\footnotetext{
${ }^{1}$ As Betti [1] puts it "This idea can be found everywhere in Bolzano's writings, starting from his early mathematical works".
} 
tools needed for a definition of being fully and immediately less complex. In order to avoid any confusion, let us present these two notions and then explain why they do not work in the grounding framework. This will also shed light on the features required from a notion of being fully and immediately less complex that is appropriate for grounding.

Definition 4.1. The complexity of a formula $\mathrm{A}, \mathrm{cm}(A)$, is inductively defined in the following way:

$$
\begin{aligned}
& \text { - } c m(p)=0 \\
& \text { - } c m(\neg A)=c m(A)+1 \\
& \text { - } c m(A \circ B)=c m(\neg(A \circ B))=c m(A)+c m(B)+1^{2}
\end{aligned}
$$

Definition 4.2. A is a subformula of B if, and only if, one of the following holds:

- $A=B$

- $B=\neg C$ and $A$ is a subformula of $C$

- $B=(C \circ D)$ and $\mathrm{A}$ is a subformula of $C$ or a subformula of $D$

Given these two definitions it seems straightforward to define the notion of being completely and immediately less complex in the following way.

Definition 4.3. In the classical logical framework, a multiset $M$ is completely and immediately less complex than a formula $C$ if, and only if:

- if $C=\neg B$, than $M=\{B\}$,

- if $C=B \circ D$, than $M=\{B, D\}$

Thus a multiset $M$ is completely and immediately less complex than a formula $C$ of complexity $n$ if, and only if, it contains all and only those subformulas of $C$ whose sum of complexities is $n-1 .^{3}$

The question now is: does Definition 4.3 work in the grounding framework? In order to answer this question, let us point out the virtues of this Definition before turning to its problems. As for the virtues, in the case of (certain) conjunctions and disjunctions, it seems to work fine. (Let us call this virtue $\mathbb{V}$.) Consider for example the formula $p \wedge q$. According to Definition 4.3 the multiset $\{p, q\}$ is completely and immediately less complex than $p \wedge q$; since $p, q$ are standardly taken to be the complete and immediate formal grounds of $p \wedge q$, this is precisely what we would like to have.

As for the problems, these are three. The first (we will call it $\mathbb{P} 1$ ) concerns conjunction and disjunction. Consider indeed a formula of the form $(p \wedge q) \wedge r$.

\footnotetext{
${ }^{2}$ From now on $\circ=\wedge, \vee$.

${ }^{3}$ It is straightforward to notice that both the "completely" and the "immediately" features of the notion of being completely and immediately less complex have been taken into account.
} 
Then it seems that we would like to claim that, not only the multiset $\{p \wedge q, r\}$ is completely and immediately less complex than $(p \wedge q) \wedge r$, but that also each of the multisets $\{q \wedge p, r\},\{r \wedge p, q\},\{p \wedge r, q\},\{q \wedge r, p\},\{r \wedge q, p\}$ is completely and immediately less complex than $(p \wedge q) \wedge r$. Of course Definition 4.3 does not allow us to draw such a conclusion.

The other two problems linked with Definition 4.3 concern negation. Consider for example the formulas $A$ and $\neg \neg A$ : $A$ is generally considered (see $[7,12,20])$ as the complete and immediate formal ground of $\neg \neg A$, thus it also needs to be completely and immediately less complex than $\neg \neg A$; however, according to Definition 4.3 , it is $\neg A$, and not $A$, that is completely and immediately less complex than $\neg \neg A$. (We will call this problem $\mathbb{P} 2$.) A similar situation arises with $\neg(A \vee B): \neg A, \neg B$ are generally considered (see [7, 12, 20]) as the complete and immediate formal grounds of $\neg(A \vee B)$, thus they also need to be completely and immediately less complex than $\neg(A \vee B)$; instead, according to our definition, it is $A \vee B$ to be fully and immediately less complex than $\neg(A \vee B)$. (We will call this problem $\mathbb{P} 3$.)

Given this situation, we can conclude that Definition 4.3, which arises from Definitions 4.1 and 4.2, does not work in the grounding framework. Thus we need an alternative notion that has the same virtue $\mathbb{V}$ of Definition 4.3, but that, at the same time, overcomes problems $\mathbb{P} 1-\mathbb{P} 3$. In order to achieve the desired result, we will proceed in three steps (that are analogous to those that we have just seen in the classical framework): we will introduce a new way of counting the complexity of a formula $A$ that is adequate for the grounding framework; then, we will explain what counts as a subformula $A$ of a formula $B$ in the grounding framework. Finally, by exploiting the first two notions, we will define the notion of being completely and immediately less complex in the grounding framework.

Let us start by presenting our measure of complexity. We give its formal definition and then we explain the ideas that motivate it.

Definition 4.4. The g-complexity of a truth $\mathrm{A}, \operatorname{gcm}(A)$, is defined in the following way:

$$
\begin{aligned}
& -\operatorname{gcm}(p)=\operatorname{gcm}(\neg p)=0 \\
& \text { - } \operatorname{gcm}(A \circ B)=\operatorname{gcm}(\neg(A \circ B))=\operatorname{gcm}(A)+\operatorname{gcm}(B)+1 \\
& \text { - } \operatorname{gcm}(\neg \neg A)=\operatorname{gcm}(A)+1
\end{aligned}
$$

The main difference between our measure of complexity, that we call $g$ complexity for grounding complexity, and the one standardly adopted in classical logic concerns the value assigned to negation, so let us try to explain this point in detail.

In classical logic one usually deals with two truth values, truth and falsity. The possibility of using the falsity as a truth value has a consequence for our way of evaluating the complexity of a formula. Consider a simple formula that starts with a negation, like $\neg p$; of this formula we say that it has complexity 
$p+1$, since a logically less complex way of conveying that $\neg p$ is true is to say that $p$ is false. Consider for example the sentence "it is not raining" is true. An equivalent, though logically less complex, way to claim that "it is not raining" is true is to say that "it is raining" is false. Thus the complexity of "it is not raining" is equal to that of "it is raining" +1 .

In the grounding framework, things are different; in the grounding framework only truths count and falsehoods do not play any role at all. It is then natural that this has a consequence for the way we evaluate the complexity of a formula, that indeed from now on will be called the complexity of a truth. In the grounding framework, if we consider a truth like $\neg p$, it is no longer the case that there exists a logically less complex way of conveying the same thing that this truth conveys, since it is no longer the case that we can use the value falsity. This implies that $\neg p$ no longer has complexity $p+1$, but it has the same complexity as $p$. Consider again the example "it is not raining" is true. In the grounding framework there is no logically less complex way of conveying the same thing; indeed "it is not raining" is as atomic as "it is raining" and thus they have the same complexity value 0 .

Analogous reasoning can be applied to the complexity of more complex truths like $\neg(A \wedge B)$ or $\neg(A \vee B)$. Even in these cases, since the value falsity does not have any role, we can no longer claim, as in the classical case, that their complexity is equivalent to the complexity of $A \wedge B+1$ and $A \vee B+$ 1 , respectively; instead, their complexity will be the same as the complexity of $A \wedge B$ and $A \vee B$, respectively.

Let us then move to the case of double negation. In case of double negation, the negation counts since $\operatorname{gcm}(\neg \neg A)=\operatorname{gcm}(A)+1$. The argument behind this way of counting double negation is once more the same. Consider the sentence "it is not the case that it is not raining" is true. The less logical complex way of saying the same thing is obtained by simply taking "it is raining" is true. Thus the complexity of "it is not the case that it is not raining" is equal to that of "it is raining" +1 .

We thus have a measure of complexity which seems adequate for the grounding framework. In order to formulate a notion of subformula which is adequate for the grounding framework - from now on we will call this type of subformula subtruth, to distinguish it from the classical one - we need two further notions: the notion of the converse of a truth $D$, denoted by $D^{*}$, and the relation $\cong$.

Let us start by introducing the notion of the converse of a truth.

Definition 4.5. The converse of a truth $D$, written $D^{*}$, is defined in the following way

$$
D^{*}= \begin{cases}\neg^{n-1} E, & \text { if } D=\neg^{n} E \text { and } \mathrm{n} \text { is odd } \\ \neg^{n+1} E, & \text { if } D=\neg^{n} E \text { and } \mathrm{n} \text { is even }\end{cases}
$$

The notion of converse of a truth is quite simple to grasp; indeed by considering a truth $A$ and its converse $A^{*}$, we are taking into account two truths that only differ by one occurrence of a negation symbol but have the same gcomplexity. So for example $p$ and $\neg p$ can be taken as a truth and its converse: 
they both have the same g-complexity 0 , but one is the negation, or the converse, of the other. The same holds for $\neg \neg \neg p$ and $\neg \neg p$ : they have the same g-complexity 1 but the latter has one negation less than the former (and so is its converse). As we will see briefly, the notion of converse of a truth happens to be very useful in the definition of the relation $\cong$ and in the definition of the notion of subtruth.

Let us now pass to the relation $\cong$ which is the second notion to introduce in order to have all the elements needed to formulate the correct definition of subformula in the grounding framework, i.e. the notion of subtruth. We firstly introduce the formal definition of $\cong$ and then we explain the intuitive idea behind it.

Consider a formula $A$. We will say that $A$ is a-c equiv to $B$ if, and only if, $A$ can be obtained from $B$ by applications of associativity and commutativity of conjunction and disjunction. ${ }^{4}$ Let us make some examples of formulas $A$ and $B$ such that $A$ is a-c equiv to $B$. If $A$ is of the form $E \wedge F$, then the formula $F \wedge E$ is $a-c$ equiv to it. If $A$ is of the form $\neg((B \vee C) \wedge(D \vee F))$ the formulas $\neg((C \vee B) \wedge(D \vee F)), \neg((B \vee C) \wedge(F \vee D)), \neg((C \vee B) \wedge(F \vee D))$ are $a-c$ equiv to it. If $A$ is of the form $((B \vee C) \vee(D \vee F))$, then the formulas $((B \vee D) \vee(C \vee F))$, $((D \vee B) \vee(F \vee C)),((B \vee F) \vee(D \vee C))$ are all a-c equiv to it.

Definition 4.6. $\mathrm{A} \cong \mathrm{B}$ if, and only if:

$\mathrm{A}$ is $a-c$ equiv to $\mathrm{B}$ or $\mathrm{A}$ is a-c equiv to $B^{*}$

Let us explain the relation $\cong$ in intuitive terms. First of all note that each truth is a-c equiv to itself, so we have that not only each truth is in the relation $\cong$ with itself, but also that each truth and its converse are in the relation $\cong$. The question now is: what kind of relation each truth entertains with itself but also with its converse? The answer is: the relation of being about, or pertaining, or concerning a given fact. Let us see this in detail. Of course given two truths $A$ and $A$, since they are identical, it is trivial to say that they also are about the same thing. But consider the more interesting case of a truth and its converse. Consider for example the truth "it is cold and it is raining" and the truth "it is not the case that it is cold and it is raining". In this case one can either focus on the fact that these truths are opposite since either "it is cold and it is raining" is true or "it is not the case that it is cold and it is raining" is true; or one can focus on the fact that these two truths share some essential features: they have the same complexity, the same atomic formulas and the same connective used in the same way and a same number of times (as explained in the previous paragraph, the presence of negation in this case does not make any difference). In sum, these two truths are about or concern the same thing: they both are about the conjunction of it is raining and it is cold irrespectively of the truth value assigned to it. This is precisely what is captured by the relation $\cong$.

Let us now pass to two truths $A$ and $B$ such that $A \neq B$ and they are $a-c$ equiv; then we have that even these two truths are in the relation $\cong$. This

\footnotetext{
${ }^{4}$ We omit the formal definition of this notion for the sake of brevity.
} 
captures the following intuitive idea. Consider the truths "it is raining and it is cold" and "it is cold and it is raining"; these two truths share some essential features: the same complexity, the same atomic truths and the same connectives used in the same way and a same number of times. Thus even of "it is raining and it is cold" and "it is cold and it is raining" we would like to say that they are about the same thing, i.e. namely the conjunction of it is raining and it is cold irrespectively to the order in which they are considered. Therefore even "it is cold and it is raining" and "it is raining and it is cold" are in the relation $\cong$.

Finally we have the case of two truths $A$ and $B$ such that $A \neq B$ and $A$ is $a-c$ equiv to $B$ and $\mathrm{A}$ and $B^{*}$ are in the relation $\cong$. In order to understand this case, we can easily adapt the explanation proposed for the other ones.

We have thus finished introducing the relation $\cong$. We now have all the elements to introduce our notion of subtruth, which is the analogue of the notion of subformula in the grounding framework.

Definition 4.7. A is a subtruth of B if, and only if, one of the following holds:

$-A \cong B$

- $B \cong \neg \neg C$ and $A$ is a subtruth of $C$,

- $B \cong(C \circ D)$ and $\mathrm{A}$ is a subtruth of $C$ or a subtruth of $D$.

In order to construct the notion of subtruth in an adequate way, we have done our best to imitate the definition of the notion of subformula, by adapting each of the concepts present and specific to the notion of subformula and to classical logic to the grounding framework. The first item of the definition of subformula states that, if $A=B$, then $A$ is a subformula of $B$. The first item of the definition of subtruth is built analogously but with the substitution of the notion of identity with the notion of $\cong$, that looks like the grounding counterpart of the identity. This yields: if $A \cong B$, then $A$ is a subtruth of $B$. This implies that not only $A$ is a subtruth of itself, but also $A^{*}$ is a subtruth of $A$, and also any truth $C$, together with its converse, such that $C$ is associatively and commutatively equivalent to $A$ is a subtruth of it.

Let us pass to the second item. The second item of the definition of subformula states that if $A$ is a subformula of $B$, then $B=\neg C$ and $A$ is a subformula of $C$. The second item of the notion of subtruth is built analogously via the following substitutions: as before identity is substituted with the relation $\cong$; the formula $\neg C$ is substituted by the formula $\neg \neg C$ because, as we have explained previously, in the grounding framework the negation counts as such only when there are two; finally, the notion of subformula is substituted by the notion of subtruth. We thus have: if $B \cong \neg \neg C$ and $A$ is a subtruth of $C$, then $A$ is a subtruth of $B$. This implies that given a truth $\neg \neg C$, not only $C$ is a subtruth of $\neg \neg C$, but also $C^{*}$ is a subtruth of $\neg \neg C$, and so is any truth $D$, together with its converse, such that $D$ is associatively and commutatively equivalent to $C$.

The parallel between the third items of the definition of subformula and the definition of subtruth is easily drawn in the light of what we have said for the 
previous two. Therefore, we have a notion of subtruth that seems adequate for the grounding framework; we can use it to define the notion of being completely and immediately less complex in the grounding framework.

Definition 4.8. In the grounding framework, a multiset $M$ is completely and immediately less complex than a truth $C$, if, and only if:

$$
\begin{aligned}
& \text { - if } C \cong \neg \neg B \text {, then } M=\{B\} \text { or } M=\left\{B^{*}\right\} \\
& \text { - if } C \cong(B \circ D) \text {, then } M=\{B, D\} \text { or } M=\left\{B^{*}, D\right\} \text { or } M=\left\{B, D^{*}\right\} \text { or } \\
& \\
& \quad M=\left\{B^{*}, D^{*}\right\} \text {. }
\end{aligned}
$$

Thus a multiset $M$ is completely and immediately less complex than a truth $C$ of complexity $n$ if, and only if, it contains all and only those subtruths of $C$ whose sum of complexities is $n-1 .^{5}$

We finally have the notion of being completely and immediately less complex in the grounding framework. This notion has been built in several steps, each of which has been deeply motivated. So we hope that Definition 4.8 does not come as a surprise but, instead, looks like the natural conclusion of all we have said in the last paragraphs.

Let us end the section by (i) giving some examples of truths that are completely and immediately less complex than another truth, (ii) checking whether the notion introduced in Definition 4.8 meets all the desiderata that we have enumerated in Section 3.

Let us start with task (i). We will give examples that are also useful to understand the role of the notion of converse of a truth and the relation $\cong$ in the definition of the notion of being completely and immediately less complex. Let us start by the truth $\neg p \wedge \neg q$; the multisets of truths that are completely and immediately less complex than $\neg p \wedge \neg q$ are $\{\neg p, \neg q\},\{p, \neg q\},\{\neg p, q\},\{p, q\}$. Consider now the truth $\neg \neg p \wedge \neg \neg q$; the multisets of truths that are completely and immediately less complex than $\neg \neg p \wedge \neg \neg q$ are $\{\neg \neg p, \neg \neg q\},\{\neg \neg \neg p, \neg \neg q\}$, $\{\neg \neg p, \neg \neg \neg q\},\{\neg \neg \neg p, \neg \neg \neg q\}$. Note that it is thanks to the notion of converse of a truth that we can indicate in an uniform way the truths that are immediately and completely less complex of $\neg p \wedge \neg q$ and $\neg \neg p \wedge \neg \neg q$. In order to obtain such truths, we should in one case erase a negation, and in the other case add a negation: the operator ${ }^{*}$ takes both these cases into account.

Consider now the truth $\neg \neg((p \wedge q) \wedge r)$. Amongst the multisets of truths that are completely and immediately less complex than $\neg \neg((p \wedge q) \wedge r)$, there are: $\{(p \wedge q) \wedge r\},\{\neg((p \wedge q) \wedge r)\}$, but also $\{(q \wedge p) \wedge r\},\{\neg((q \wedge p) \wedge r)\}$, $\{(q \wedge r) \wedge p\},\{\neg((q \wedge r) \wedge p)\},\{(q \wedge p) \wedge r\},\{\neg((q \wedge p) \wedge r)\}$. The role of the relation $\cong$ is to allow all these different multisets.

Let us now turn to task (ii): we should check whether the notion of being completely and immediately less complex as we introduced it in Definition 4.8 has the virtue $\mathbb{V}$ and overcomes the problems $\mathbb{P} 1$ - $\mathbb{P} 3$ that we have illustrated in

\footnotetext{
${ }^{5} \mathrm{As}$ it was the case in the classical framework, it is straightforward to notice that both the "completely" and the "immediately" features of the notion of being completely and immediately less complex have been taken into account.
} 
Section 3. As for the virtue $\mathbb{V}$, this is satisfied; to see this, consider a truth like $p \wedge q$. According to Definition 4.8, amongst the multisets which are completely and immediately less complex than $p \wedge q$ there is $\{p, q\}$ (the others are $\{\neg p, q\}$, $\{p, \neg q\}$ and $\{\neg p, \neg q\})$ and this is precisely what we wanted.

Let us now pass to problems $\mathbb{P} 1$ - $\mathbb{P} 3$ and let us start by analyzing problem $\mathbb{P} 1$. Such a problem has been overcome by Definition 4.8: indeed, amongst the multisets that are completely and immediately less complex than a truth like $(p \wedge q) \wedge r$, there are the following ones $\{p \wedge q, r\},\{q \wedge p, r\},\{p \wedge r, q\},\{r \wedge p, q\}$, $\{q \wedge r, p\},\{r \wedge q, p\}$ and this is precisely what we wanted. Let us now move to problems $\mathbb{P} 2$ and $\mathbb{P} 3$, which both concerned negation. On the one hand, we have that, according to our Definition 4.8, amongst the multisets that are completely and immediately less complex than a truth like $\neg \neg p$ there are both $\{p\}$ and $\{\neg p\}$. Thus problem $\mathbb{P} 2$ has been overcome. On the other hand, we have that, according to our Definition 4.8, amongst the multisets that are completely and immediately less complex than a truth like $\neg(p \wedge q)$ there is $\{\neg p, \neg q\}$ (the others are $\{p, q\},\{\neg p, q\}$ and $\{p, \neg q\})$. Thus problem $\mathbb{P} 3$ has also been solved. So all our desiderata have been satisfied; this could be taken as an additional sign of the adequateness of the proposed notion of being completely and immediately less complex for the grounding framework.

\section{The paradigmatic case of disjunction (or why we need another ingredient to define formal grounding)}

We have seen some salient features of the notion of formal grounding that partly come from the literature and partly have been developed in this paper. More precisely, in the previous sections, we have argued that the notion of classical derivability and the notion of being completely and immediately less complex are two necessary conditions for complete and immediate formal grounding. The question now is whether they also are sufficient. In this section we will show that they are not. In the next section we will introduce the final missing ingredient in the definition of formal grounding.

In order to show why the notions of classical derivability and being less complex are necessary but not sufficient for the definition of formal grounding, we will use the paradigmatic case of disjunctive truths. Let us then consider a disjunctive truth like $A \vee B$ and suppose that $A$ is true. $A$ is then a ground for $A \vee B$. But is $A$ the complete ground for $A \vee B$ ? The answer would seem to depend on the truth of $B$. If $B$ is true, then $A$ and $B$ together are the complete grounds of $A \vee B$. And if $B$ is false? In this case, $A$ would seem to constitute the complete grounds of $A \vee B$ : but this is only because $B$ is false. Indeed, as just noted, if $B$ were true, $A$ would no longer constitute the complete grounds of $A \vee B$ (it would merely be a partial ground). Hence, in the case where $B$ is false, it still has a role to play in determining the grounds of $A \vee B$ : its falsity ensures that or is a condition for $A$ to be the complete grounds for $A \vee B$. To 
capture this role, we shall say that $A$ is the complete grounds for $A \vee B$ under the robust condition that $B$ is false, or equivalently, we will say that $A$ is the complete grounds for $A \vee B$ under the robust condition that the converse of $B$ is true. Thus, according to this analysis of disjunctive truths, in order to give the complete and immediate grounds of $A \vee B$, a distinction between grounds and robust conditions is required: indeed either both disjuncts are the complete grounds of the disjunctive truth, or if only one of them is, this can only happen under the condition that the converse of the other is true.

This treatment of disjunctive truths seems reasonable and adequate. Let us make a concrete example of the complete and immediate grounds of a disjunctive truth. Let us consider the sentence "I take the umbrella or the coffee is good". According what we have said up to now, the complete and immediate grounds of "I take the umbrella or the coffee is good" are the following:

- either "I take the umbrella" and "the coffee is good";

- or "I take the umbrella" under the robust condition that "the coffee is not good";

- or "the coffee is good" under the robust condition that "I do not take the umbrella".

Each of the two disjuncts "I take the umbrella" and "the coffee is good" has always a role to play in determining the complete grounds of "I take the umbrella or the coffee is good". If it is true, it belongs to the complete grounds of "I take the umbrella or the coffee is good". If it is false, then its falsity in itself is a condition for the other disjunct to be a complete ground for "I take the umbrella or the coffee is good": after all, if "the coffee is good" were true, "I take the umbrella" would no longer be the complete grounds for "I take the umbrella or the coffee is good" (and vice-versa for if "I take the umbrella" were true).

Let us now go back to our analysis of grounding and let us suppose, for the sake of the argument, that grounding is defined by means of classical derivability and the notion of being completely and immediately less complex. Let us call this definition $D-C$ def. Let us try to understand whether $D-C$ def can properly take into account the disjunctive case alongs the lines set out above. More precisely, let us try to understand whether $D-C$ def is powerful enough to render the distinction between grounds and robust conditions that we have just introduced and that is specific to the disjunctive case. To answer these questions, let us consider the disjunctive truth "I take the umbrella or the coffee is good" and let us suppose that "I take the umbrella" and "the coffee is not good" are both true. Then, according to $D-C$ def, we have that both "I take the umbrella" and "the coffee is not good" are the complete and immediate grounds of "I take the umbrella or the coffee is good". Indeed from "I take the umbrella" and "the coffee is not good" "I take the umbrella or the coffee is good" is derivable, and "I take the umbrella" and "the coffee is not good" are completely and immediately less complex than "I take the umbrella or the coffee 
is good" according to our Definition 4.8 of being completely and immediately less complex. Thus, if we define formal grounding just in terms of derivability and complexity, we cannot make any distinction between grounds and robust conditions. Moreover, we obtain the mistaken conclusion that "the coffee is not good" is a ground of "I take the umbrella or the coffee is good". This means that $D-C$ def is not powerful enough to render the subtle distinctions specific to the grounding framework; this means that we are lacking an ingredient in our definition of complete and immediate formal grounding.

\section{A definition of the notion of complete and im- mediate formal grounding}

In this section we will introduce and discuss the third and last ingredient of our definition of complete and immediate formal grounding. Our intuitive idea can be explained as follows. Let us take as our starting point the claim that in a grounding relation the consequent is strictly connected to its grounds. This sounds like a claim which is rather unproblematic (the reader can find similar claims in $[8,13])$, but also quite vague. The question is then that of making it more precise, i.e. the question is then that of clarifying this idea of strict connection. In order to clarify the idea of strict connection, we will use the concept of variation; we will indeed say that in a grounding relation the consequent varies together with each modification of its grounds. This new claim seems to present the same characteristics of the previous one: on the one hand, it sounds acceptable (the idea of explaining a connexion within a variation is certainly not new, see $[14,16]$ ), but in need of further clarification. Indeed, if we really want to understand what grounding is about, we need to specify what kind of modifications the grounds might have and what exactly it means for the consequent to vary with its grounds. In order to clarify these issues, let us recall an important point that has already been emphasized several times: in a grounding framework we only deal with truths and grounding is a relation amongst truths. In the light of this, the answers to our questions seem easier. Firstly, we might want to say that the grounds can only be modified in two ways: either they are true or the negation of each of them is true; secondly, we might want to assert that for the consequent to vary with its grounds is the same as for the consequent to track the truth of its grounds: if the grounds are true, then the consequent is true too; but also, if the negation of each ground were true, the negation of the consequent would be true too. To put it as Nozick [16] did, though for grounding rather than for knowledge:

To ground a sentence is to have that sentence track the truth of its reasons. Grounds and their consequence are connected in a particular way, the latter tracks the truth of the former.

We have thus clarified the idea of a consequent varying together with its grounds by means of the idea of a consequent tracking the truth of its grounds. 
Let us now make a further and final step and present the formal counterpart of this concept of tracking the truth. For this, we will use the concept of classical derivability. Therefore, not only, like we have already seen, we will say that in a grounding relation the consequent is derivable from its grounds, but also, and this is the third and last ingredient of the definition of formal grounding, that the negation of the consequent is derivable from the negation of each of its ground. By specifying both these types of derivability, we finally have a proper account of the notion of formal grounding. Indeed, it is only by specifying both these types of derivability that we have the full formal counterpart of the idea of strict connection that lies at the heart of the notion of (formal) grounding.

Let us try to write down these intuitions in a more formal way. For this let us denote robust conditions with the notation $[C]$, where $C$ is a formula of the language $\mathcal{L}^{c}$; moreover we will adopt the following convention $\neg(M):=$ $\{\neg B \mid B \in M\}$. We can now formulate the following definition.

Definition 6.1. For any consistent multiset of formulas $C \cup M$, we say that under the robust condition $\mathrm{C}$, the multiset $M$ completely and immediately formally grounds $A,[C] \mathrm{M} \mid \sim \mathrm{A}$, if and only if:

- $M \vdash A$

- $C, \neg(M) \vdash \neg A$

- $C \cup M$ is completely and immediately less complex than $A$, in the sense of being completely and immediately less complex given in Definition 4.8.

Under the robust condition $\mathrm{C}$, the multiset $M$ completely and immediately formally grounds $A$ if, and only if, (i) $A$ is derivable from $M$ - we will call this item positive derivability; (ii) $\neg A$ is derivable from $\neg(M)$ plus $C$ - we will call this item negative derivability; (iii) $C \cup M$ is completely and immediately less complex than $A$ - we will call this item complexity. The notion of complexity describes the grounding hierarchy in which truths are organized; in particular, the notion of being completely and immediately less complex gives an exhaustive description of each step of this hierarchy. The notion of positive and negative derivability tell us which truths in each step of this hierarchy are linked by a grounding relation.

We finally have the definition of complete and immediate formal grounding. This definition have been built in several steps, each of which has been justified. So we hope that Definition 6.1 does not come as a surprise but, instead, looks like the natural conclusion of all we have said in the previous sections. We will end up the section by testing our Definition 6.1 on the problematic case of disjunction. The capacity to deal with this case could be taken as a sign of the adequateness of the definition itself.

Let us consider again the truth "I take the umbrella or the coffee is good" and, for the sake of brevity, let us formalize it with $p \vee q$. According to what we have said in the previous section, we would like the complete and immediate formal grounds of $p \vee q$ to be: 
- either $p$ and $q$,

- or $p[\neg q]$,

- or $q[\neg p]$.

Let us check whether this is the case according to Definition 6.1. Let us start from the case of $p$ and $q$; these are indeed the complete and immediate grounds of $p \vee q$ according to Definition 6.1: from $p$ and $q, p \vee q$ is derivable; from $\neg p$ and $\neg q, \neg(p \vee q)$ is also derivable; and finally $p, q$ are completely and immediately less complex than $p \vee q$. Let us now pass to $p[\neg q]$; according to Definition $6.1 p$ is the complete and immediate ground of $p \vee q$ under the robust condition $\neg q$. From $p, p \vee q$ is derivable; from $\neg p$ and $\neg q, \neg(p \vee q)$ is also derivable and, finally, $p$ and $\neg q$ are completely and immediately less complex than $p \wedge q$. As for the case $q[\neg p]$, this works analogously to the previous one. Thus Definition 6.1 seems to respond to all our expectations in the case of disjunction and this, as already emphasized, might be taken as a (first) sign of the adequateness of our definition. We will use the next section to expose other interesting characteristics of our notion of grounding.

\section{Comparisons between our approach and other approaches to the notion of grounding}

We will dedicate this section to the analysis of the differences between our account of the notion of complete and immediate formal grounding and the one that can be found in three articles ${ }^{6}$ recently published by Correia [7], Fine[12], Schnieder [20]. This will shed light, not only on the originality of our approach, but also, and more importantly, on its most noteworthy features.

The differences between our approach to the notion of grounding and the one adopted by the aforementioned authors are of three kinds: the first concerns the case of disjunction and negation of conjunction; the second concerns the case of truths that are associatively and commutatively equivalent, and the third concerns the case of negation. We will analyze them one by one.

Let us start by the case of disjunction and negation of conjunction. In order to analyze this case, we will mainly focus on disjunctive truths, leaving aside truths that have the form of negation of conjunction. We will do this for the sake of brevity, knowing that everything that can be said about disjunction can also be said about negation of conjunction.

Let us then consider once more the disjunctive truth "I take the umbrella or the coffee is good". According to [7, 12, 20], the full and immediate grounds of "I take the umbrella or the coffee is good" are the following:

- either "I take the umbrella" and "the coffee is good"

\footnotetext{
${ }^{6}$ Each author presents his own analysis of the notion of grounding, which is different from the analysis given by the other two authors. Nevertheless, these three approaches have enough common points to be gathered together and compared with our own approach.
} 
- or "I take the umbrella"

- or "the coffee is good"

Thus their treatment of the disjunctive case is quite distant from the one presented in Section 5. This difference is simply due to a difference of the notion of grounding that the approaches want to analyze. While our approach focuses on the notion of complete and immediate grounding, their approach concentrates on the notion of full and immediate grounding; the two notions, though similar, are not the same (see Section 3); in particular, a little reflection suffices to realize that it is precisely in the case of disjunction (and negation of conjunction) that their diversity comes to the fore. We can thus conclude that there is not tension amongst the two results.

Let us now pass to examine the second difference between our approach and that of Correia, Fine and Schnieder. In order to explain this difference, we will focus and use truths that have a conjunctive form. However, we note that this is not limitative in any way: everything that will be said about this type of truths can easily be adapted to any other type of propositional truth. Let us then start our analysis. Anyone who is acquainted with the contemporary literature on grounding $[7,12,20]$ will acknowledge that, given a truth of the form $A \wedge B,\{A, B\}$ is the unique full and immediate ground of $A \wedge B$. What we claim in this paper is something different. We indeed affirm that, given a truth of the form $A \wedge B$, not only $\{A, B\}$ is a complete and immediate ground of $A \wedge B$, but so is any multiset $\{C, D\}$ such that $C \wedge D$ is associatively and commutatively equivalent to $A \wedge B$. Since the difference between what is said in the current literature and our approach is quite significant, we feel compelled to further justify and explain our position.

For this let us focus on an even more particular example; let us consider the truth $(p \wedge q) \wedge r$. According to the way we have defined our notion of complete and immediate formal grounding (Definition 6.1), not only the multiset $\{p \wedge q, r\}$ is a complete and immediate formal ground of $(p \wedge q) \wedge r$, but also each of the multisets $\{q \wedge p, r\},\{p \wedge r, q\},\{r \wedge p, q\},\{r \wedge q, p\}$ and $\{q \wedge r, p\}$ is. $^{7}$ As already explained in the paragraph above, this is a quite big departure from the current literature, since, according to Correia, Fine and Schnieder, only the multiset $\{p \wedge q, r\}$ is the full and immediate ground of $(p \wedge q) \wedge r$. The task is then that of explaining why each of the following multisets $\{q \wedge p, r\},\{p \wedge r, q\}$, $\{r \wedge p, q\},\{r \wedge q, p\}$ and $\{q \wedge r, p\}$ needs to be considered as a complete and immediate ground of the truth $(p \wedge q) \wedge r$. In order to develop this task, we will only concentrate on the multiset $\{q \wedge p, r\}$; what is said about this multiset can easily be repeated for the remaining ones.

Let us then consider $\{q \wedge p, r\}$ and the truth $(p \wedge q) \wedge r$ and let us analyze their relationship in the grounding framework. When taken together these truths undoubtedly stand in a grounding relation; in particular, it seems natural to claim that the multiset $\{q \wedge p, r\}$ is a ground of $(p \wedge q) \wedge r$. This grounding relation appears so straightforward that it would be up to the one who denies it to argue

\footnotetext{
${ }^{7}$ Let us note that in $[6]$ we can find a similar idea for the logic of conceptual grounding.
} 
against it; the issue is therefore that of understanding what type of grounding relation is involved. We recall that we only have four possibilities: either it is a relation of complete (or full) and immediate grounding, or a relation of complete (or full) and mediate grounding, or of partial and immediate grounding or of partial and mediate grounding. In the next paragraphs we will try to identify the appropriate option.

Let us start by the distinction complete - partial, which is the easiest one. No matter how one defines this distinction, either precisely in terms of complete - partial or in terms of full - partial, the conclusion is the same: $\{q \wedge p, r\}$ cannot but be a complete or full ground of $(p \wedge q) \wedge r$. Indeed in $\{q \wedge p, r\}$ each of the atomic truths that compose $(p \wedge q) \wedge r$ can be found again and nothing is missing. Thus we have to rule our the option partial grounding. Let us now then move to the distinction immediate-mediate. Even in this case the situation does not appear as too complicated. We are indeed in a formal grounding framework where each grounding step corresponds to the introduction of a new connective. But $\{q \wedge p, r\}$ and the truth $(p \wedge q) \wedge r$ precisely differ for one connective, namely a conjunction; thus only one grounding step separates them and so it is straightforward to say that they stand in an immediate grounding relation.

Given what we have just seen, we can draw the conclusion that, whatever approach to grounding one adopts, one is committed to accept that the multiset $\{q \wedge p, r\}$ is a complete (or full) and immediate ground of $(p \wedge q) \wedge r$; actually we are committed to accept that each of the multisets $\{q \wedge p, r\},\{p \wedge r, q\}$, $\{r \wedge p, q\},\{r \wedge q, p\}$ and $\{q \wedge r, p\}$ is a complete (or full) and immediate ground of $(p \wedge q) \wedge r$. The situation is so because of two things; on the one hand, it cannot be denied that between the multisets $\{q \wedge p, r\},\{p \wedge r, q\},\{r \wedge p, q\},\{r \wedge q, p\}$ and $\{q \wedge r, p\}$ and the truth $(p \wedge q) \wedge r$ there is a grounding relation. But, once this is acknowledged, the only grounding relation this grounding relation can be is the one of complete (or full) and immediate grounding. Therefore this is an important feature of the concept of grounding that has not been emphasized before. Our account, instead, properly takes it into account and adequately treats it.

Let us now move to the analysis of the third and final difference between our approach and the one presented in the papers $[7,12,20]$. This difference is quite easy to explain. Consider a truth of the form $\neg(A \vee B)$ : while in our account a complete and immediate ground of this truth is the multiset $\left\{A^{*}, B^{*}\right\}$, according to $[7,12,20]$ the full and immediate ground of this truth is the multiset $\{\neg A, \neg B\}$. Thus, once more, the two accounts differ and it seems necessary to better understand such a difference.

For this, consider the question of which of the followings are the complete and immediate grounds of the truth "it is not the case that it is not raining or it is not cold": "it is raining" and "it is cold" on the one hand, and "it is not the case that it is not raining" and "it is not the case that it is not cold" on the other hand. In front of this situation we guess that whoever has an intuitive idea of what grounding is would answer "it is raining" and "it is cold": these truths are indeed less complex than the others and of the grounded truth and thus represent the best option for fulfilling the role of complete and immediate 
grounds.

Let us now show how our approach takes into account these intuitions. For the sake of brevity, let us formalize "it is not the case that it is not raining or it is not cold" with $\neg(\neg p \vee \neg q)$. According to our account, the complete and immediate grounds of $\neg(\neg p \vee \neg q)$ are $(\neg p)^{*}$ and $(\neg q)^{*}$, i.e. $p$ and $q$. Indeed from $p$ and $q, \neg(\neg p \vee \neg q)$ is derivable, from $\neg p$ and $\neg q, \neg \neg(\neg p \vee \neg q)$ is derivable, and $p$ and $q$ are completely and immediately less complex than $\neg(\neg p \vee \neg q)$. According to our approach, and contrary to [7, 12, 20]'s approach, $\neg \neg p$ and $\neg \neg q$ are not the grounds of $\neg(\neg p \vee \neg q)$ : indeed, despite the fact that from $\neg \neg p$ and $\neg \neg q$, $\neg(\neg p \vee \neg q)$ is derivable, and from $\neg \neg \neg p$ and $\neg \neg \neg q, \neg \neg(\neg p \vee \neg q)$ is derivable (and thus positive and negative derivability are satisfied), $\neg \neg p$ and $\neg \neg q$ are not less complex than $\neg(\neg p \vee \neg q)$; actually, according to our measure of g-complexity, they are more complex. Thus, our approach, and in particular our measure of g-complexity which has been carefully introduced and well-motivated in Section 4 , seem more compelled to formalize some very natural intuitions concerning the use of negation in the grounding framework. We consider this characteristic to be an important contribution of our notion of grounding.

\section{Conclusions}

In this paper we have focussed on the notion of complete and immediate formal grounding and we have provided a definition for this notion. The two key ingredients of the definition are: the classical notion of derivability and a notion of complexity that has been built specifically for the grounding framework. Thanks to this definition, we have developed an original and accurate analysis of several emblematic grounding cases.

We think that the results of this paper should serve as a basis for several different directions of future research. Here we outline three of them, that we deem as the most significant ones. First of all, it would be important to adapt our definition of complete and immediate grounding to the cases of complete and mediate, partial and immediate and partial and mediate grounding. Secondly, it would be interesting to extend our analysis of grounding from the propositional case to the first-order case. Finally and most crucially, it would be imperative to use the results of this paper for developing a logic of grounding that could potentially differ from those that already exist in the literature.

\section{References}

[1] A. Betti. Explanation in metaphysics and Bolzano's theory of ground and consequence. Logique et analyse, 211:281-316, 2010.

[2] B. Bolzano. Theory of Science: A Selection, with an Introduction. D. Riedel, Dordrecht, December 1973.

[3] Bernard Bolzano. Contributions to a more well founded presentation of 
mathematics. In William Bragg Ewald, editor, From Kant to Hilbert : A source book in the foundations of mathematics, pages 176-224. Oxford University Press, Oxford, 1996.

[4] E. Casari. Matematica e verità. Rivista di Filosofia, 78(3):329-350, 1987.

[5] F. Correia. Grounding and truth-functions. Logique et Analyse, 53(211):251-79, 2010.

[6] F. Correia. The impure logic of conceptualistic grounding. In Recent work on the logic of grounding Workshop, Oslo, 2014.

[7] F. Correia. Logical grounds. Review of Symbolic Logic, 7(1):31-59, 2014.

[8] F. Correia and B. Schnieder. Grounding: an opinionated introduction. In F. Correia and B. Schnieder, editors, Metaphysical grounding, pages 1-36. Cambridge University Press, Cambridge, 2012.

[9] C. Daily. Scepticism about grounding. In F. Correia and B. Schnieder, editors, Metaphysical grounding, pages 81-100. Cambridge University Press, Cambridge, 2012.

[10] L. de Rosset. What is weak ground? Essays in Philosophy, 14(1):7-18, 2013.

[11] K. Fine. Some puzzles of ground. Notre Dame Journal of Formal Logic, 51(1):97-118, 2010.

[12] K. Fine. Guide to ground. In F. Correia and B. Schnieder, editors, Metaphysical grounding, pages 37-80. Cambridge University Press, Cambridge, 2012.

[13] K. Fine. The pure logic of ground. Review of Symbolic Logic, 25(1):1-25, 2012.

[14] D. Lewis. Causation. Journal of Philosophy, 70(3):556-567, 1973.

[15] J. E. Litland. On some counterexamples to the transitivity of grounding. Essays in Philosophy, 14(1):19-32, 2013.

[16] R. Nozick. Philosophical explanations. Harvard University press, 1981.

[17] F. Paoli. Bolzano e le dimostrazioni matematiche. Rivista di Filosofia, LXXXIII:221-242, 1991.

[18] A. Rumberg. Bolzano's theory of grounding against the background of normal proofs. Review of Symbolic Logic, 6(3):424-459, 2013.

[19] B. Schnieder. A puzzle about 'Because'. Logique et Analyse, 53(4):317-343, 2010. 
[20] B. Schnieder. A logic for 'Because'. The Review of Symbolic Logic, 4(03):445-465, 2011.

[21] J. Sebestik. Logique et mathematique chez Bernard Bolzano. J. Vrin, 1992.

[22] A. Tatzel. Bolzano's theory of ground and consequence. Notre Dame Journal of Formal Logic, 43(1):1-25, 2002.

[23] A. S. Troelstra and H. Schwichtenberg. Basic Proof Theory. Cambridge University Press, 1996. 